\title{
Lightness constancy: Ratio invariance and luminance profile
}

\author{
Alessandro Soranzo \\ University of Teesside, Middlesbrough, England \\ Alessandra Galmonte \\ University of Verona, Verona, Italy \\ AND \\ Tiziano Agostini \\ University of Trieste, Trieste, Italy
}

\begin{abstract}
The term simultaneous lightness constancy describes the capacity of the visual system to perceive equal reflecting surfaces as having the same lightness despite lying in different illumination fields. In some cases, however, a lightness constancy failure occurs; that is, equal reflecting surfaces appear different in lightness when differently illuminated. An open question is whether the luminance profile of the illumination edges affects simultaneous lightness constancy even when the ratio invariance property of the illumination edges is preserved. To explore this issue, we ran two experiments by using bipartite illumination displays. Both the luminance profile of an illumination edge and the luminance ratio amplitude between the illumination fields were manipulated. Results revealed that the simultaneous lightness constancy increases when the luminance profile of the illumination edge is gradual (rather than sharp) and homogeneous (rather than inhomogeneous), whereas it decreases when the luminance ratio between the illumination fields is enlarged. The results are interpreted according to the layer decomposition schema, stating that the visual system splits the luminance into perceived lightness and apparent illumination components. We suggest that illumination edges having gradual and homogeneous luminance profiles facilitate the luminance decomposition process, whereas wide luminance ratios impede it.
\end{abstract}

Simultaneous lightness constancy is the phenomenon whereby equal reflecting surfaces are perceived as having the same lightness despite lying in different illumination fields. This phenomenon constitutes a problem for vision science, since visual objects are perceived by means of the light rays reflected from the surfaces to the retina. The amount of light reflected by the surfaces reaching the retina (i.e., luminance) is the product of the incident light and the reflectance of the surfaces. Therefore, equal reflecting surfaces placed in different illumination fields project different amounts of light to the retina. Nevertheless, under many conditions, surfaces sharing the same reflectance are perceived as having the same lightness, although differently illuminated. How can the visual system recognize the reflectance equality among surfaces despite their projecting different amounts of light to the retina?

To recognize this equality, it is necessary for the visual system to distinguish the different illumination intensities in the visual image. In this regard, it has been proposed that to achieve the simultaneous lightness constancy, the visual system has to detect which, among the luminance edges in the image, are the illumination edges (Gilchrist, 1988). According to this view, one way to investigate simultane- ous lightness constancy is to find out which cues help the visual system to individuate illumination changes. Two of these cues are investigated in the present research: the ratio invariance property and the gradual luminance transition.

The ratio invariance property refers to a physical property of the illumination edges. According to Gilchrist (1988), the nature of the intersection where an illumination edge crosses a reflectance edge can determine whether the first will actually be perceived as a change in illumination. This intersection, indeed, owns the ratio invariance property; that is, the luminance ratio between regions under different illumination intensities remains the same when an illumination edge crosses them. Therefore, in order for the simultaneous lightness constancy to occur, an illumination edge has to cross at least one reflectance edge, so as to create the ratio invariance cue. If, on the other hand, an illumination edge does not cross any reflectance edge, the simultaneous lightness constancy should not occur, and physically equal surfaces should be perceived as having different lightness. Experimental evidence has shown that this is what actually happens. Specifically, Gilchrist (1988) has shown that when the intersection between an illumination and a reflectance edge

A.Soranzo, a.soranzo@tees.ac.uk 
is hidden from view, surfaces sharing the same reflectance are perceived to be different in lightness, leading, therefore, to a simultaneous lightness constancy failure.

Concerning the other illumination cue, the gradual luminance transition, Hering (1920) observed that a shadow covering a homogeneous surface appears as a dark stain if the gradual luminance transition at its edge is masked by a black ink. In other words, when the gradual luminance transition at the shadow edges is masked, the underlying surfaces differ in perceived lightness, leading, again, to a simultaneous lightness constancy failure. The observation that gradual luminance transitions help the visual system to individuate illumination changes has been confirmed by other studies (Agostini \& Galmonte, 1997, 2002; Kardos, 1934; MacLeod, 1947). The outcomes of these researches have induced the authors to conclude that gradual luminance transitions favor the percept of a change in the illumination intensity. In these studies, however, the gradual luminance transitions were the only illumination cue in the visual scene, since the ratio invariance property was never maintained. Because of that, gradual luminance transitions in conjunction with the ratio invariance property have never been considered together as a cue favoring the occurrence of the simultaneous lightness constancy. Hence, an unresolved question is, Do gradual luminance transitions help the visual system to achieve the simultaneous lightness constancy even when the ratio invariance property is maintained?

To answer this question, we compared the effects of gradual and sharp luminance transitions on simultaneous lightness constancy by using a display in which the ratio invariance property was always preserved. Therefore, since the gradual luminance transitions were not the only illumination cue in the image, we created critical conditions for testing their effect on simultaneous lightness constancy.

\section{EXPERIMENT 1}

In this experiment, the effects of illumination edges that had a gradual luminance profile on simultaneous lightness constancy were compared with the effects of illumination edges that had a sharp luminance profile. The congruency of the illumination edge profile was also manipulated. It could be either congruent (i.e., homogeneously gradual or homogeneously sharp) or incongruent (i.e., partially gradual and partially sharp). Finally, we manipulated the luminance ratio amplitude between regions separated by the illumination edge.

\section{Method}

Observers. Twenty-six volunteer observers participated in this experiment. All had normal or corrected-to-normal vision and were naive with regard to the experimental design.

Apparatus and Stimuli. The stimuli were generated by a Macintosh Quadra 840/AV computer and were presented on a calibrated CRT Trinitron monitor $(1,280 \times 1,024$ pixels $) .{ }^{1}$ The screen of the monitor was vertically divided into two halves by a luminance edge. Then two additional rectangles were positioned at the center of these two halves. The luminance ratio between the two areas on the left and the corresponding areas on the right was the same. Under these conditions, with the ratio invariance property preserved, the four areas could be perceived as two surfaces (referred to as contextual and mediating backgrounds) under two illumination intensities: the illuminated and the shadowed fields. The portion of the luminance edge dividing the screen vertically that crossed the contextual background was the contextual edge, whereas the portion of the same edge crossing the mediating background was the mediating edge. On the shadowed field, there was another surface, the standard, having a fixed luminance, whereas on the illuminated field, there was the target, whose luminance was adjustable from the computer console. We manipulated three experimental variables: (1) congruency of the illumination edge, (2) mediating edge profile, and (3) luminance ratio amplitude.

The congruency variable had two levels: (1) congruent (the mediating and the contextual edges shared the same profile) and (2) incongruent (the mediating and the contextual edges had different profiles). The mediating edge profile variable was also dichotomous: (1) gradual (the luminance profile at the mediating edge was gradual) and (2) sharp (the luminance profile at the mediating edge was sharp). For the luminance ratio amplitude variable, the luminance ratio between the illuminated and the shadowed fields was manipulated; it could be $2: 1,10: 1$, and 30:1. Altogether, there were 12 stimuli $(2 \times 2 \times 3)$.

Figure 1A depicts the displays; ${ }^{2}$ Figure 1B indicates the luminance in $\mathrm{cd} / \mathrm{m}^{2}$ (the question mark indicates that the luminance of the target was randomly assigned by the software at the beginning of each trial); and Figure 1C shows both the size of each area of the displays in degrees (the width of the gradual transition, not indicated in the figure, was $1^{\circ}$ ) and the names of the two portions of the illumination edge.

Procedure. The observers viewed the stimuli, presented in random order, in a darkened room at a distance of $57 \mathrm{~cm}$ from the monitor. They were instructed to match the lightness of the target patch on the illuminated field to the corresponding standard patch on the shadowed field, by using the plus and minus keys of the keyboard. Pressing the space bar signaled that a satisfactory match was achieved. At that point, the target luminance was recorded, and the next trial began. The luminance of the target was set to a random value at the beginning of each trial. In order to obtain a lightness match, the observers were asked to make the target patch "look as if it were cut from the same piece of paper as the standard." The observers performed two matches for each of the 12 stimuli, so they provided 24 matches. Each display was left on the screen as long as needed to produce the match. The whole session lasted about $20 \mathrm{~min}$.

\section{Results and Discussion}

Mean ratings are made comparable by an index that can be defined as the Thouless luminance ratio (TLR). The TLR is the same as the Thouless (1931) ratio but uses luminance values directly, instead of transforming them into reflectance. This is, therefore, very useful for measuring lightness constancy degree in research in which the reflectance values are laborious to derive.

According to Fechner's (1889) law, luminance values are first transformed into log units. Then, by following Thouless's (1931) proposal, the following formula is obtained:

$$
\text { TLR }=\frac{\log (\mathrm{PSE})-\log (L)}{\log (R)-\log (L)},
$$

where PSE is the point of subjective equality, $L$ is the luminance of the standard, and $R$ is the theoretical luminance value corresponding to perfect constancy.

Finally, by means of simple mathematical transformations, the following manageable index is obtained:

$$
\mathrm{TLR}=\frac{\log (\mathrm{PSE} / L)}{\log (\text { ratio })},
$$


A

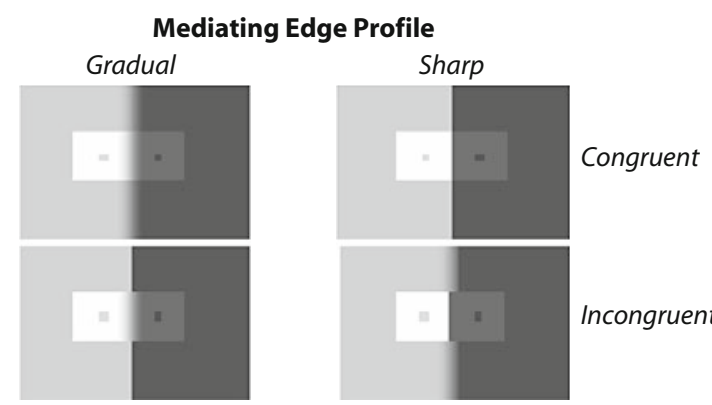

B

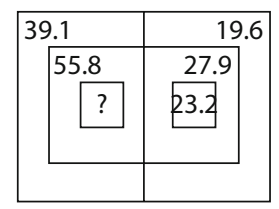

2:1

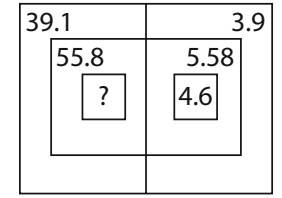

$10: 1$

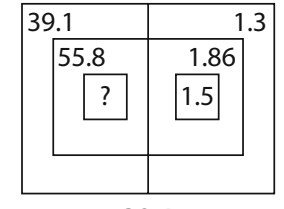

$30: 1$

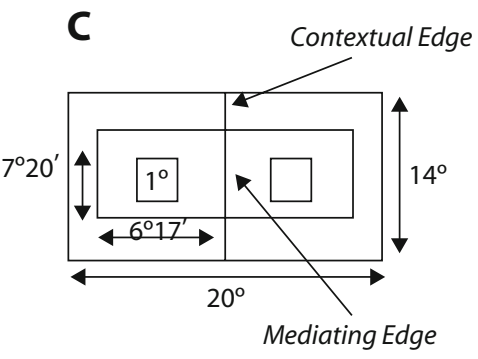

Figure 1. (A) Stimuli in the first experiment (10:1 luminance ratio amplitude only). They are displayed according to their level for the mediating edge profile variable (columns) and congruence variable (rows). (B) Luminance values (in $\mathrm{cd} / \mathrm{m}^{2}$ ) for the three levels of the luminance ratio amplitude variable. (C) Stimuli size in degrees of visual angle. The width of the gradual transition (not shown in the figure) was equal to $1^{\circ}$.

where ratio is the luminance ratio between the illumination fields.

Mean index-linked values and the standard errors are shown in Figure 2.

A three-way repeated measures ANOVA, performed on the index-linked values, revealed a significant effect of the three experimental variables $(p<.001)$, whereas the interactions among them were not significant.
As can be observed in Figure 2, for all the levels of the luminance ratio amplitude variable, the constancy index is closer to one (i.e., the constancy degree is higher) when the luminance profile at the mediating edge is gradual rather than sharp. Furthermore, the degree of constancy improves when the illumination edge profile is congruent-that is, when the mediating and contextual edges share the same profile.

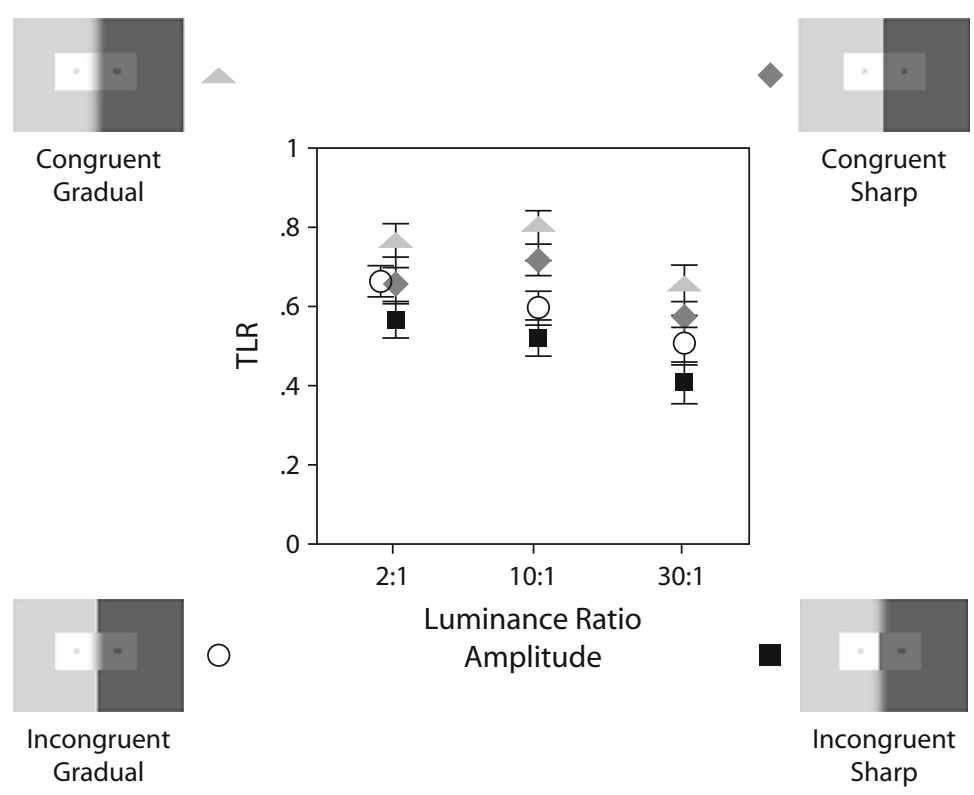

Figure 2. Mean index-linked values (Thouless luminance ratio [TLR]) in the first experiment. Bars represent standard errors. 
The least squares means analysis revealed a significant difference among all the conditions for both the mediating edge profile and the congruency variables $(p<.001)$. Specifically, it emerged that the constancy degree was higher in the congruent-gradual than in the congruentsharp condition $[t(25)=5.2, p<.01]$.

These results indicate that gradual luminance transitions improve the constancy degree even when the ratio invariance property is preserved. Furthermore, this effect seems to be independent of the luminance ratio amplitude between the illumination fields.

Figure 2 shows also that for all the levels of the congruency and mediating edge profile variables, the constancy degree was lower in the 30:1 condition rather than in the other conditions. The least squares means analysis indicated a significant difference between the $30: 1$ and $10: 1$ conditions and between the $30: 1$ and $2: 1$ conditions $[t(25)=4.58, p<.01$, and $t(25)=5.75, p<.01$, respectively], whereas the difference between the 2:1 and 10:1 conditions was not significant. This suggests that there is no correspondence between the constancy degree and the luminance ratio amplitude: When the luminance ratio between an illuminated and a shadowed field is $30: 1$, the constancy degree decreases, as compared with conditions having a smaller ratio. From this experiment, however, it is not possible to understand whether the constancy failure occurring in the 30:1 condition was due to the wide ratio or to the low luminance intensities in shadow. These two variables, indeed, changed at the same time. Therefore, a second experiment was performed in which both the luminance ratio amplitude and the luminance intensities were controlled.

\section{EXPERIMENT 2}

The experimental displays in the first experiment differed for both the luminance intensities in shadow and the luminance ratio amplitude between the illumination fields. In order to identify which one of these two factors was more effective in determining the constancy failure registered in the 30:1 condition, in the second experiment we controlled for both factors.

\section{Method}

Observers. Ten volunteer observers participated in this experiment. All had normal or corrected-to-normal vision and were naive with regard to the experimental design. None of them had participated in the first experiment.

Apparatus and Stimuli. The apparatus was the same as that in the first experiment. As in the first experiment, the stimuli were simulations of a scene under two different illumination intensities. Hence, there were mediating and contextual backgrounds vertically crossed by a luminance edge. There was also a standard, in shadow, and a target in light. The sizes of the display areas were the same as those in the first experiment (see Figure 1C). There were two independent variables; the first was the absolute luminance values with three levels.

1. High luminance, which was identical to the 10:1 display in the first experiment.

2. Low luminance, in which all the luminance values were reduced by one third, as compared with the high-luminance condition. In this way, the luminance ratio amplitude between the two illumination fields was equal to $10: 1$ as in the high-luminance condition, but all the luminance values were lowered.

3. Large ratio, in which the luminance values in the illuminated field were the same as those in the high-luminance condition, whereas those in the shadowed field were the same as those in the low-luminance condition. The luminance ratio amplitude between the two fields was 30:1 (this display was, therefore, identical to the 30:1 display in the first experiment).
A

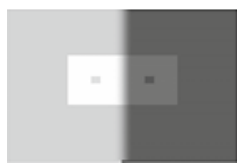

Gradual

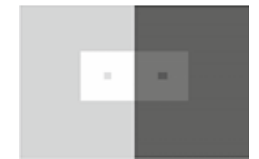

Sharp

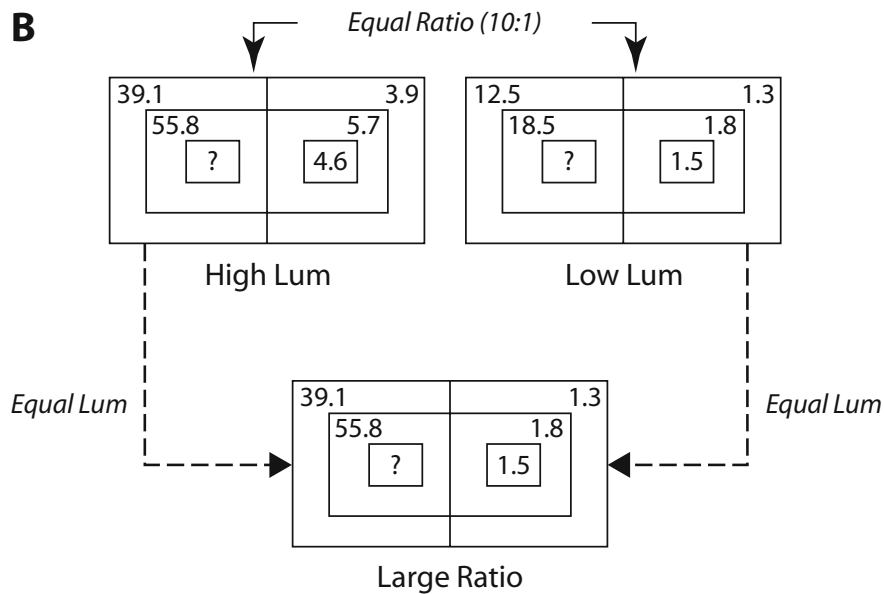

Figure 3. (A) Levels of the illumination edge profile variable. (B) $\mathrm{Lu}$ minance (in $\mathrm{cd} / \mathrm{m}^{2}$ ) of the absolute luminance values variable. 
The second independent variable was the illumination edge profile with two levels: (1) gradual (the luminance profile of the illumination edge was gradual) and (2) sharp (the luminance profile of the illumination edge was sharp).

Figure $3 \mathrm{~A}$ shows the experimental conditions for the illumination edge profile, and Figure 3B shows the luminance, in $\mathrm{cd} / \mathrm{m}^{2}$, of the displays.

In total, there were six stimuli $(3 \times 2)$.

Procedure. The procedure was the same as that in the first experiment.

\section{Results and Discussion}

Mean ratings were transformed with the same TLR index as that used in the first experiment (see the Results section for the first experiment). A two-way repeated measures ANOVA, performed on the index-linked values, revealed a significant effect of both the absolute luminance values and the illumination edge profile variables $[F(2,9)=4.82$, $p<.05$, and $F(1,9)=17.19, p<.01$, respectively]. The interaction between them was not significant. Figure 4 depicts the mean index-linked values and the standard errors in the second experiment.

The least squares means comparison performed on the absolute luminance values variable revealed a significant difference between the high-luminance and the large-ratio conditions and between the low-luminance and the largeratio conditions $[t(9)=2.60, p<.05$, and $t(9)=2.77$, $p<.05$, respectively]. There was no significant difference between the high- and low-luminance conditions. There was a significant difference between conditions differing in luminance ratio amplitude, but no difference between the conditions differing in luminance intensities. It seems, therefore, that when the luminance ratio amplitude between the illumination fields is $10: 1$, the simultaneous lightness constancy improves, in comparison with the condition in which this ratio is $30: 1$, and this effect seems to be independent of the absolute luminance intensities.

In addition, in the second experiment, the effect of luminance profile on simultaneous lightness constancy was repli-

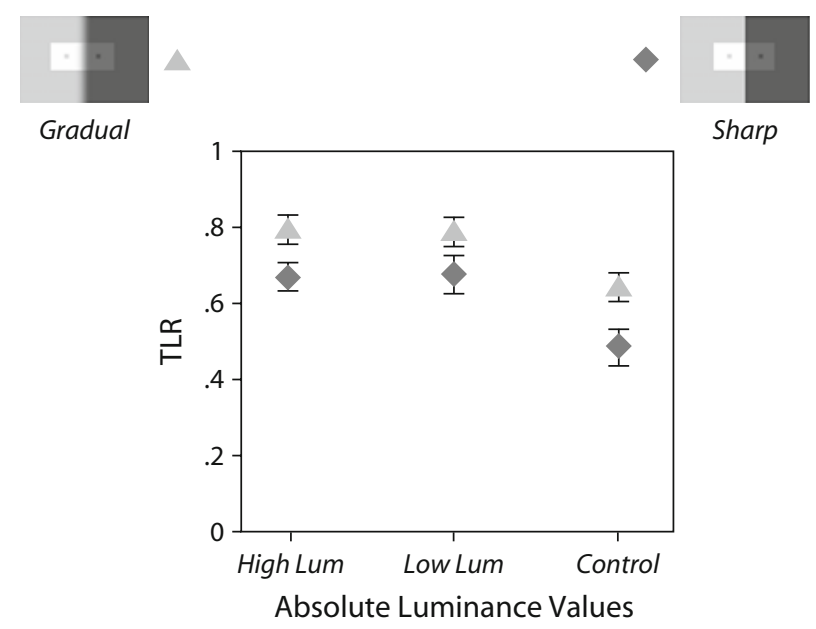

Figure 4. Mean index-linked values in the second experiment (Thouless luminance ratio [TLR]). Bars represent standard errors. cated. When the profile of the illumination edge was gradual, the simultaneous lightness constancy degree was higher, as compared with the conditions in which it was sharp.

\section{GENERAL DISCUSSION}

From our work, it emerges that in a bipartite illumination field display, lightness constancy is affected by the following factors: (1) the illumination edge luminance profile, (2) the congruency of the illumination edge, and (3) the luminance ratio amplitude between the illumination fields. These factors will be discussed separately.

\section{The Illumination Edge Luminance Profile}

The first aim of the present research was to answer the following question: Do gradual luminance transitions help the visual system to achieve simultaneous lightness constancy when the ratio invariance property is maintained? The outcomes show that, when the luminance ratio invariance property is preserved, simultaneous lightness constancy improves when the luminance profile of an illumination edge is gradual rather than sharp. It seems, therefore, that the ratio invariance property and the gradual luminance transition do cooperate to achieve a better constancy degree. In addition, this effect was found to be independent of the luminance ratio amplitude between the fields.

To interpret this outcome, we consider two theories on lightness perception, both taking into account highlevel visual processes. Most lightness theorists have accepted the high-level viewpoint, suggested first by Koffka (1935), that the retinal image is decomposed into separate components. However, there are two types of decomposition schemas: the layer type and the framework type.

The layer decomposition schema states that the visual system decomposes the pattern of light intensities reaching the eyes into separate contributions: reflectance, illumination, and so on (Adelson \& Pentland, 1990; Barrow \& Tenenbaum, 1978; Beck, 1972; Bergstrom, 1977; Gilchrist, 1977, 1979; Kozaki, 1965; Musatti, 1953; Oyama, 1968; Schirillo, 1999a, 1999b).

Although this schema accounts for the simultaneous lightness constancy phenomenon, it has been argued that its attempt to explain the losses of constancy has not proven to be very effective (Gilchrist, 2005, 2006; Gilchrist et al., 1999). If the visual system were able to split the luminance into components, errors should not occur in lightness perception. However, this is not the case; in some conditions, the visual system attributes different lightness values to equal reflecting surfaces. To solve this problem, it has recently been proposed that, in those conditions, the visual system may commit an error in the layer decomposition process (Anderson \& Winawer, 2005; Gilchrist, 1988; Soranzo \& Agostini, 2004, 2006a, 2006b). This error can be named the luminance misattribution, meaning that part of the luminance that should be attributed to one component is attributed to another instead. The amount of luminance misattribution should be inversely proportional to the number and strength of illumination cues in the visual scene. 
In the displays in this research, the luminance misattribution should imply that (1) part of the standard luminance that should have been attributed to its reflectance was attributed to the illumination and/or (2) part of the target luminance that should have been attributed to the illumination was attributed to its reflectance.

Since the constancy degree was higher when the profile of the illumination edge was gradual rather than sharp, it seems that both luminance ratio invariance and gradual luminance transitions are cues facilitating the correct layer decomposition process and having additive effects.

The framework approach, however, argues against the illumination interpretation of the luminance gradients (Bressan, 2006; Gilchrist et al., 1999). This approach claims that the visual system divides the image "into contiguous regions of illumination or shadow, like states on a map" (Gilchrist, 2005, p. 331). The perceived lightness of any given surface depends mainly on its photometric relationship with the highest luminance (anchor) in the same framework.

In comparison with the layer approach, errors in lightness perception are operationalized much better: They depend on the amount of framework segregation. The more one framework is segregated by the others, the more the perceptual lightness is correctly computed.

According to this view, the role of the luminance gradients should be to increase the framework segregation, leading to an increase in lightness constancy. In the displays in the present research, both the ratio invariance and the gradual luminance transition cooperated to produce higher lightness constancy degrees. Therefore, according to the framework approach, these factors should contribute conjunctively and cumulatively to segregate the image into frameworks.

To sum up, the increase of lightness constancy degree produced by the gradual luminance transitions in the presence of the ratio invariance property can be interpreted by both of the decomposition schemas. According to the layer approach, these factors should facilitate luminance attribution to the different components; according to the framework approach, they should facilitate image segregation into frameworks.

In the next section, however, it will be shown that the effect on simultaneous lightness constancy of the congruency of the illumination edge cannot be explained by both of the decomposition schemas.

\section{Congruency of the Illumination Edge Luminance Profile}

The second major outcome of the present research is that simultaneous lightness constancy improves when the congruency of the illumination edge profile is maintained rather than altered. Specifically, the results show a better lightness constancy degree when a mediating and a contextual edge share the same luminance profile, rather than a different one. This effect is so robust that the constancy degree was higher in the congruent-sharp display than in the incongruent-gradual display. This point needs to be stressed because in the incongruent-gradual condition, the surfaces to be compared in lightness (standard and target) were separated by a gradual luminance transition, whereas in the congruent-sharp condition, they were separated by a sharp transition. Therefore, it seems that in order to achieve a better constancy degree, the congruency of the illumination edges is more effective than the type of their luminance profile.

This outcome has very important theoretical consequences because, as we have anticipated, it seems to challenge one of the decomposition schemas.

As has been stressed, the results show better constancy in the congruent-sharp rather than in the incongruentgradual condition. According to the layer decomposition schema, the degree of constancy should depend on the number and strength of illumination cues in the visual scene. Congruency of the illumination edge should be considered one of the most important illumination cues, favoring correct luminance attribution to the different components. Since in the incongruent-gradual condition this factor is absent, whereas it is present in the congruentgradual condition, the layer approach correctly accounts for the better lightness constancy degree measured when the congruency of the illumination edge is preserved.

According to the framework approach, however, in the incongruent-gradual condition, the ratio invariance property and the gradual luminance transition are both active factors in cumulatively segregating the frameworks, whereas in the congruent-sharp condition, the ratio invariance property is the sole active factor operating in the segregation. Therefore, better lightness constancy degree should occur in the incongruent-gradual than in the congruentsharp condition; but this is not what actually happens.

By comparing the results of the two incongruent conditions, another conclusion can be drawn. The outcomes reveal that the gradual luminance profile at the mediating edge increases the simultaneous lightness constancy degree, as compared with the sharp profile at this edge. This difference can be explained by taking into account the number of cues signaling that the target and the standard were in different illumination fields. Whereas in the incongruent-sharp condition, this information is carried out from the ratio invariance property only, in the incongruent-gradual condition, this information is strengthened by the presence of the gradual luminance transition at the mediating edge (only in the incongruentgradual case were the standard and the target separated by a gradual luminance transition; see Figure 1).

Hence, the layer decomposition approach explains this result too. In the next section, however, we will see that this approach too is not exempt from difficulties.

\section{Luminance Ratio Amplitude Between the Illumination Fields}

In the first experiment in this research, it emerged that when the luminance ratio amplitude between the illumination fields was equal to $30: 1$, the constancy degree decreased with respect to the $10: 1$ or 2:1 case.

Therefore, in bipartite displays, when the luminance values in shadow are reduced, a constancy failure occurs. A similar result emerged in the studies of Katz $(1911,1935)$ and Helson (1943). Both authors attributed this effect to the 
low luminance intensities in the shadowed fields in their displays. However, in the second experiment in the present research, the effects of the luminance values in shadow on the simultaneous lightness constancy were compared with those of the luminance ratio amplitude between the illumination fields. Contrary to Katz's and Helson's suggestions, we found that when the intensities in shadow were kept constant, wide luminance ratios between the illumination fields induced a lightness constancy failure.

Interpreting this result within both the theoretical decomposition schemas is tricky. The framework schema does not include, at present, the luminance ratio amplitude among frameworks as a factor affecting lightness perception. To explain this result within the framework paradigm, it should be assumed that the higher the luminance ratio between two frameworks, the lower their segregation from each other. This explanation seems, at most, counterintuitive.

On the other side, to explain this result within the layer decomposition paradigm, it has to be assumed that the relation linking the luminance ratio amplitude with lightness constancy is not a linear relation. In this regard, Beck (1972) suggested that the relation linking lightness to the apparent illumination is inversely proportional but that it varies when the absolute luminance in the visual scene is changed, leading, therefore, to a nonlinear relation. Following Beck's idea, it could be argued that the visual system correctly (or almost correctly) splits the luminance in the stimuli only within a small luminance range; beyond that range, a greater luminance misattribution should occur. This means that the visual system does not consider wide luminance ranges among illumination fields as illumination cues. This is unexpected, considering that from photometric measurements of natural scenes, it has emerged that the widest luminance ratios are produced by illumination changes, rather than reflectance ones (Brown \& Deffenbacher, 1979; Dember \& Warm, 1979; Goldstein, 1980). Therefore, one would expect that the visual system would consider wide luminance ratios as an illumination cue. Nevertheless, this seems not to be what actually happens.

This puts in evidence one of the major shortcomings of the layer approach: It is difficult to postulate a priori what the visual system will consider an illumination cue, and what it will not. The other important shortcoming of this schema is that no predictions as to what an observer actually perceives are given; it predicts only the relative lightness values, instead of their specific values. In particular, the layer approach does not include any anchor for deriving perceived lightness values. On the contrary, the anchor is one of the advantages of the framework approach. Therefore, in accordance with Gilchrist (2005), we believe that integration of the layer and the framework decomposition schemas might give rise to a more comprehensive lightness theory. One possibility is that the visual system first attributes luminance to the different components, basing this attribution on the number and strength of the illumination cues in the image; then, to the provisory lightness values resulting from this attribution, principles proposed by the framework decomposition schemas are applied. This hypothesis needs to be tested with further experiments.

\section{AUTHOR NOTE}

This research was supported by PRIN: 2007E7PHM3 to T.A. We thank Allison Sekuler, Alan Gilchrist, and two anonymous reviewers for their valuable comments and suggestions. Correspondence concerning this article should be addressed to A. Soranzo, School of Social Science and Law, University of Teesside, Middlesbrough TS1 3BA, England (e-mail: a.soranzo@tees.ac.uk).

Note-Accepted by the previous editorial team, when Thomas H. Carr was Editor.

\section{REFERENCES}

Adelson, E. H., \& Pentland, A. P. (1990). The perception of shading and reflectance (Vision and Modeling Tech. Rep. 140). Cambridge, MA: Massachusetts Institute of Technology, Department of Brain Research and Cognitive Sciences.

Agostini, T., \& Galmonte, A. (1997). Luminance gradients, perceived illumination, and lightness perception. Review of Psychology, 4, 3-6.

Agostini, T., \& Galmonte, A. (2002). A new effect of luminance gradient on achromatic simultaneous contrast. Psychonomic Bulletin \& Review, 9, 264-269.

ANDERSON, B., \& WinAwER, J. (2005). Image segmentation and lightness perception. Nature, 434, 79-83.

Barrow, H. G., \& Tenenbaum, J. (1978). Recovering intrinsic scene characteristics from images. In A. R. Hanson \& E. M. Riseman (Eds.), Computer vision systems (pp. 3-26). Orlando, FL: Academic Press.

BECK, J. (1972). Surface color perception. Ithaca, NY: Cornell University Press.

Bergstrom, S. S. (1977). Common and relative components of reflected light as information about the illumination, colour, and threedimensional form of objects. Scandinavian Journal of Psychology, 18, $180-186$

BRESSAN, P. (2006). The place of white in a world of grays: A doubleanchoring theory of lightness perception. Psychological Review, 113, 526-553.

Brown, E. L., \& Deffenbacher, K. (1979). Perception and the senses. New York: Oxford University Press.

Bruno, N. (1994). Failures of lightness constancy, edge integration, and local edge enhancement. Vision Research, 34, 2205-2214.

Dember, W. N., \& Warm, J. S. (1979). Psychology of perception. New York: Holt, Reinhart \& Winston.

Fechner, G. T. (1889). Elemente der Psychophysik (2nd ed.). Leipzig: Breitkopf \& Härtel.

Gilchrist, A. L. (1977). Perceived lightness depends on perceived spatial arrangement. Science, 195, 185-187.

Gilchrist, A. L. (1979). The perception of surface blacks and whites. Scientific American, 240, 112-123.

GiLchrist, A. L. (1988). Lightness contrast and failures of constancy: A common explanation. Perception \& Psychophysics, 43, 415-424.

GILCHRIST, A. L. (2005). Lightness perception: Seeing one color through another. Current Biology, 15, R330-R332.

Gilchrist, A. L. (2006). Seeing black and white. Oxford: Oxford University Press.

Gilchrist, A. L., Kossyfidis, C., Bonato, F., Agostini, T., CataLIOTTI, J., LI, X., ET AL. (1999). A new theory of lightness perception. Psychological Review, 106, 795-834.

Goldstein, E. B. (1980). Sensation and perception. Belmont, CA: Wadsworth.

HeLson, H. (1943). Some factors and implications of color constancy. Journal of the Optical Society of America A, 33, 555-567.

HERING, E. (1920). Grundzüge der Lehre vom Lichtsinn. Berlin: Springer Kardos, L. (1934). Ding und Schatten. Leipzig: Barth.

KatZ, D. (1911). Die Erscheinungsweisen der Farben und ihre Beeinflussung durch die individuelle Erfahrung. Zeitschrift für Psychologie, 7, 6-31.

Katz, D. (1935). The world of colour. London: Kegan Paul, Trench, Trubner.

KoFfKa, K. (1935). Principles of Gestalt psychology. New York: Harcourt, Brace \& World

KozAKI, A. (1965). The effect of co-existent stimuli other than the test 
stimulus on brightness constancy. Japanese Psychological Research, 7, 138-147.

MacLeod, R. B. (1947). The effect of "artificial penumbrae" on the brightness of included areas. In Miscellanea psychologica Albert Michotte (pp. 138-154). Louvain: Institut Superieur de Philosophie.

Musatti, C. L. (1953). Ricerche sperimentali sopra la percezione cromatica. Archivio di Psicologia, Neurologia e Psichiatria, 14, 541-577.

Oyama, T. (1968). Stimulus determinants of brightness constancy and the perception of illumination. Japanese Psychological Research, 10, 146-155.

SCHIRILlo, J. A. (1999a). Surround articulation: I. Brightness judgments. Journal of the Optical Society of America A, 16, 793-803.

SCHIRILlo, J. A. (1999b). Surround articulation: II. Lightness judgments. Journal of the Optical Society of America A, 16, 804-811.

SorAnzo, A., \& Agostini, T. (2004). Impossible shadows and lightness constancy. Perception, 33, 1359-1368.

Soranzo, A., \& Agostini, T. (2006a). Does perceptual belongingness affect lightness constancy? Perception, 35, 185-192.

Soranzo, A., \& Agostini, T. (2006b). Photometric, geometric, and perceptual factors in illumination-independent lightness constancy. Perception \& Psychophysics, 68, 102-113.

Thouless, R. H. (1931). Phenomenal regression to the "real" object. British Journal of Psychology, 22, 1-30.

\section{NOTES}

1. The use of the CRT monitor in lightness studies has pros and cons. The impoverished degree of ecological validity of the CRT method is compensated for by the flexibility in controlling the spatial distribution of luminances (Bruno, 1994). Recently, the use of the CRT method in lightness and color investigations has extensively increased, reinforced by the fact that outcomes coming from studies adopting this technology have been perfectly in line with outcomes emerging from similar studies that adopted surfaces reflecting light.

2. For the sake of brevity, Figure 1A represents only the 10:1 level of the luminance ratio amplitude variable.

(Manuscript received August 15, 2006; revision accepted for publication October 27, 2008.) 